\title{
LA-UR-98-3202
}

Approved for public release; distribution is unlimited.

\section{CONF-980733_- Title: THE DEVELOPMENT OF A VIRTUAL HEAT BATH FOR CALORIMETERS}

\section{Author(s):}

M. M. Pickrell, D. S. Bracken, and C. R. Rudy

Submitted to:

1998 INMM

Naples, FL USA

July 26-30, 1998

(FULL PAPER)

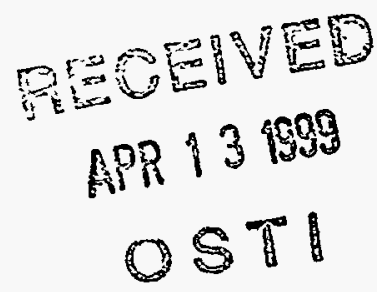

DISTRIBUTION OF THIS DOCUMENT IS UHLRATED $P$

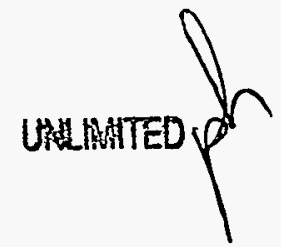

MASTER

\section{Los Alamos}

Los Alamos National Laboratory. an affirmative action/equal opportunity employer, is operated by the University of Califomia for the U.S. Department of Energy under contract W-7405-ENG-36. By acceptance of this articie. the publisher recognizes that the U.S. Government retains a nonexclusive. royaltyfree license to publish or reproduce the published form of this contribution, or to allow others to do so. for U.S. Government purposes. Los Alamos National Laboratory requests that the publisher identify this article as work performed under the auspices of the U.S. Department of Energy. Los Alamos National Laboratory strongly supports academic freedom and a researcher's nght to publish; as an institution. however, the Laboratory does not endorse the viewpoint of a publication or guarantee its technical correctness. 


\section{DISCLAIMER}

This report was prepared as an account of work sponsored by an agency of the United States Government Neither the United States Governmeat nor any ageacy thereof, nor any of their employees, makes any warranty, express or implied, of assumes any legal liability or responsibility for the securacy, completerest, of usefulness of any information, apparatus, product, or process diselosed, of represents that its use would not iniringe privately owned sights. Reference berein to any specific commercial produch, process, or serviee by trade anme, tradernarte, inzoufaeturer, or otherwise does not aecessarily constitute or imply its endorsement, recommendation, or favoring by the United States Goyernmeat of any agency thereof. The views and opinions of authors expressed herein do not secessarily state or reflect those of the United States Governmeat or any agency thereof. 


\section{DISCLAIMER}

Portions of this document may be illegible in electronic image products. Images are produced from the best available original document. 


\title{
THE DEVELOPMENT OF A VIRTUAL HEAT BATH FOR CALORIMETERS
}

\author{
M. M. Pickrell, D. S. Bracken, and C. R. Rudy \\ Los Alamos National Laboratory \\ Los Alamos, New Mexico 87545 USA
}

\begin{abstract}
All existing calorimeter systems for sensitive nuclear assay employ a heat bath surrounding the sample chamber. The purpose of the heat bath is to maintain a constant temperature so that a fixed temperature difference is maintained across the thermal resistance of the calorimeter. Present calorimeter systems all employ an active, feedback-controlled system to maintain a fixed temperature. An alternative would be to allow the heat-bath temperature to change, to measure it, and to compensate the assay for this change. Two significant observations make this approach possible: 1) the effect on the measurement of a temperature change in the heat bath is differential in form and 2) temperature measurement systems are very accurate when measuring differences in temperature (either in time or between two locations). From these observations, we have developed a virtual heat-bath compensation system. The control theory and results will be presented.
\end{abstract}

\section{INTRODUCTION AND BACKGROUND}

Present calorimeters use feedback-controlled temperature stabilization systems to control the temperature of the calorimeter heat bath. The heat bath provides a stable and accurate temperature reference against that the temperature rise of the sample can be compared. The heat bath is essential for only one reason - to eliminate ambient temperature fluctuations on the time scale of the equilibration of the calorimeter - otherwise, these would introduce a bias error in the measurements. The feedback systems have other valuable benefits, though. By maintaining the heat bath at a constant temperature, the calorimeter equilibration time can be shortened. A stable thermal gradient does not need to be fully established to achieve an equilibrium assay, particularly in the case of "servo.controlled" calorimeters.

However, feedback-control systems have several significant problems, some of which have only been manifested with new, highly sensitive, fiber-optic systems. The first problem with feedbackcontrolled systems is the possibility of violating the Nyquist stability criterion and becoming unstable. In extreme cases, the feedback system could be completely unstable with no final equilibrium, but the more common case would be that the equilibrium would be under damped and would oscillate toward its final value. This effect could measurably degrade precision and extend assay time. Another effect of the instability would be to preclude prediction by any rational algorithm, which further increases assay time. Any rational prediction algorithm will depend on the appropriate placement of the system function poles, which is satisfied when the gain, $G \leq 1$, and the system is causal. These conditions are violated for even a marginally unstable system.

Feedback systems are problematic for other reasons as well. Fundamental to feedback systems is the fact that the control precision is inversely proportional to the loop gain. To obtain tight control of the temperature, a high-gain loop is required. The high gain pushes the system toward instability (violating the Nyquist criterion) and also requires large temperature changes in the thermal controller. These can introduce thermal gradients and fluctuations in the heat bath, which are another source of assay error. 
One approach that might maintain the benefits of feedback control but eliminate the problems would be to use compensation rather than feedback control. Compensation works by sensing the heat-bath temperature and correcting the measurement for this effect, rather than attempting to control the temperature. The feedback loop would be eliminated (or strongly decoupled) and replaced with a compensation algorithm. Because there would be no (or a decoupled) feedback loop, there would be no instability. Therefore the system would remain critically damped. It would be possible to develop a rational prediction algorithm, and no thermal fluctuations would be introduced from this source.

Another important benefit to compensation is that it would improve effective heat-bath performance. Present feedback systems are limited by the heat-bath heat capacity and the Nyquist stability criterion to a certain level of temperature precision. A compensation system would not be limited by these effects and might further improve the effective heat-bath precision.

\section{THE APPROACH}

The concept of a heat-bath compensation system has been explored both theoretically and experimentally. Both sets of results will be shown here. The results clearly demonstrate that heatbath compensation is possible and can improve calorimeter performance.

The compensation of the calorimeter heat bath requires a temperature sensor at the interface between the heat bath and the calorimeter thermal resistance. This location is typical of the thermal control surface for servo-control calorimeters and is also the putative location for the reference leg of differential temperature measurements. This sensor detects heat-bath temperature changes at the thermal resistance point. The calorimeter would have a differential temperature measurement across this thermal resistance, which could be a resistive-wire bridge or a Michelson interferometer. The essential physics of this idea is the fact that the calorimeter only responds to changes in heat-bath temperature, its differential followed by a decaying exponential or sum of exponentials. We exploit this property because it does not matter what the absolute ambient temperature is, only changes in the heat-bath temperature.

The advantage of the single-element temperature sensor used in this mode is that it naturally provides a temperature reference. At some time, defined as $t=0$, the sensor has to be at some temperature (the exact value is unimportant). That temperature serves as a reference temperature and the sensor measures temperature changes relative to that value. 
The second temperature measurement should be wound in close contact with the reference leg of the differential measurement so they are at the same temperature. The output of the compensation measurement is processed using the signalprocessing algorithm discussed below. The result is then used to compensate the calorimeter assay. In operation, the differential measurement would make the calorimetry assay; it would measure the temperature difference across the thermal resistance (sample container to heat bath). The single-element measurement would measure changes or fluctuations in the heat-bath temperature. The results of the single measurement would be processed using the signal processing discussed below to compensate the differential measurement and reduce the error effect from temperature fluctuations in the heat bath. Figure 1 shows the proposed interferometer design with windings for both temperature sensors. The method for analyzing the data from the compensation measurement is discussed in the next section.

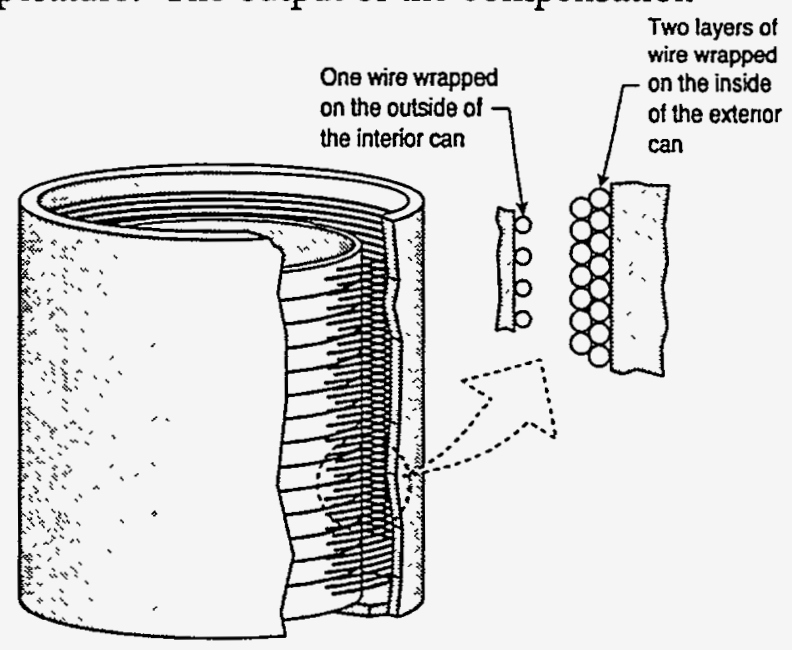

Fig. 1. The proposed calorimeter design. The Michelson interferometer has two windings, one on the outside of the sample container and one on the inside of the heat-bath surface. The F-P interferometer has a single winding also on the inside of the heat-bath surface.

\section{THE HEAT BATH}

Ideally, a calorimeter comes to full equilibrium and the temperature of the heat bath is unimportant. The calorimeter measures the temperature difference across the thermal resistance, which depends only on the heat source of the sample and the thermal resistance. However, even when the system has achieved equilibrium, there can be temperature fluctuations near the inside surface of the heat bath. These temperature changes will immediately affect the differential temperature measurement across the thermal resistance, and will remain in effect until the system reaches a new equilibrium. Although the calorimeter can operate at any ambient temperature, these temperature changes will modify the measurement. Significantly, these thermal fluctuations can occur from ambient temperature changes or from the equilibrium process of the calorimeter with a sample.

The effect of the temperature changes decays away on thermal equilibration time scales. Figure 2 illustrates this problem for a simple case - a step-function change in heat-bath temperature. A step-function change in temperature causes an immediate change in the measured temperature difference (because the heat bath temperature is different), but this decays away with the thermal equilibration time.

The more general problem is that there are many temperature fluctuations and the system can never fully reach equilibrium. To demonstrate this effect, we created a simple simulation for a simple thermal transfer system to test this issue, consisting of a simple, first-order equation for the thermal equilibration. The simulation was used to create the plots in Fig. 2. The results show a single decaying exponential in response to a step change in heat-bath temperature. When the thermal fluctuations are a more general spectrum, the resulting temperature error would be similar to that shown in Fig. 3. Figure 3 uses the same simulation and plots the temperature fluctuations for the calorimeter assuming a random temperature change superposed with a sinusoid. This function was chosen to test the simulation. Figure 3 illustrates that the calorimetry system never achieves 
equilibrium in the presence of continued temperature changes. These plots are theoretical, but closely model real system behavior.

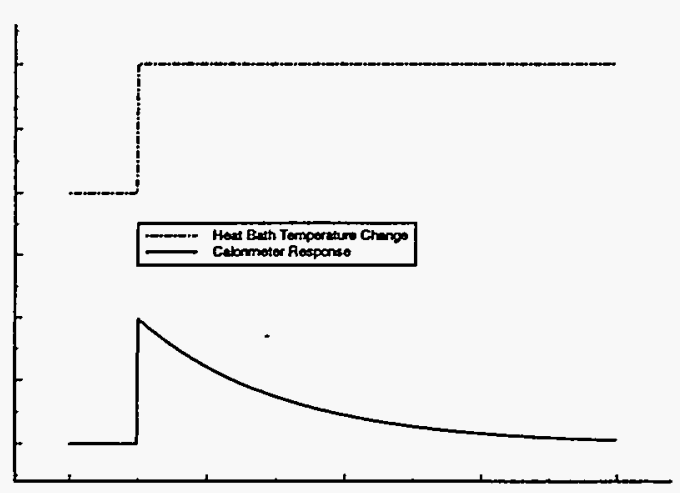

Fig. 2. Idealized plot of heat bath temperature change (top plot) and calorimeter response (bottom plot). The step function change in heat bath temperature decays away with the thermal equilibration time.

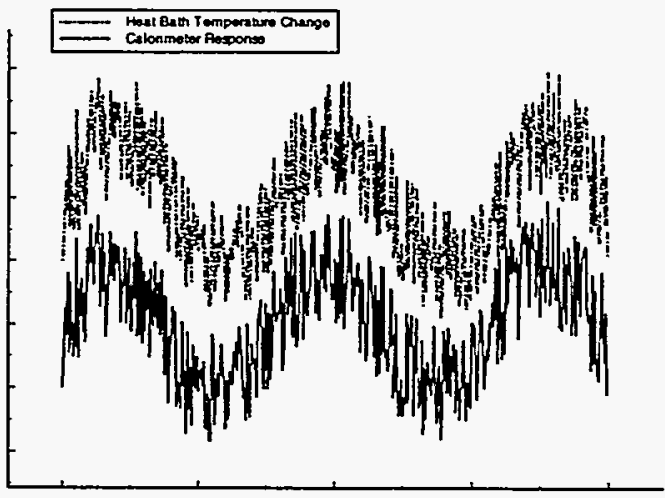

Fig. 3. The top plot is the simulated heat-bath temperature change, consisting of a random signal superposed with a sinusoid. The bottom plot is the calorimeter response.

\section{TIME DOMAIN ANALYSIS OF THE COMPENSATION MEASUREMENT}

The results in Figs. 2 and 3 are from a simple thermal equilibrium simulation using a finite difference equation. Starting from the basic result that the system response to a step function is a decaying exponential, we can develop a time-dependent correction for the calorimeter. The compensation measurement is used to correct for the fluctuations also seen by the differential measurement. As shown in Fig. 2, a step function in the heat-bath temperature produces the decaying exponential response. These two time functions are related by the convolution of the calorimeter equilibration system function:

$\underline{\text { Decaying Exponential }}=\underline{\text { System Function }}$ convolved with Step Function. The equation is:

$$
\mathrm{u}(t) e^{-\alpha t}=\mathrm{u}(t) \otimes H(t)
$$

This response can be more easily analyzed using Laplace transforms. Taking the Laplace transform of both sides of 2:

$$
\frac{1}{s+\alpha}=\frac{1}{s} \cdot H(s)
$$

where $\mathrm{H}(\mathrm{s})$ is the Laplace transform of $\mathrm{H}(t)$, and $s \equiv j \omega$ :

$$
H(s)=\mathcal{L}[H(t)]
$$

The transfer function, $\mathrm{H}(\mathrm{s})$, can be solved for with simple algebra:

$$
H(s)=\frac{s}{s+\alpha}
$$

An important property of the transfer function in Eq. 4 is that it is explicitly differential. The " $s$ " in the numerator is the Laplace differentiation operator: $s \mathrm{X}(s)=L\left[\frac{d \mathrm{X}(t)}{d t}\right]$. This result is important 
because it means that an absolute temperature measurement of the heat bath is unnecessary. It is only necessary to accurately measure the change in heat-bath temperature, relative to an arbitrary value. The compensation measurement provides precisely this measurement.

There are several approaches that can be taken to convert the compensation temperature measurement according to Eq. 4 so that it can correct the differential temperature measurement for thermal fluctuations in the heat bath. The signal could be Laplace transformed, multiplied by the transfer function, $\mathrm{H}(\mathrm{s})$, and the result is inverse Laplace transformed. Alternatively, $\mathrm{H}(\mathrm{s})$ could be converted to a digital filter that could be used to "filter" the compensation signal. The filtered signal would then be subtracted from the differential measurement. Perhaps the easiest approach is to inverse Laplace transform $\mathrm{H}(\mathrm{s})$ and then convolve this result with the compensation signal. Since the calorimeter system makes multiple discrete measurements, the result should be in the discretetime domain. The inverse Laplace transform of $\mathrm{H}(\mathrm{s})$ must then be converted to discrete time. Alternatively, this entire derivation could be repeated explicitly in the discrete-time domain using the Z-transform. Both methods will be shown here. The inverse Laplace transform of $\mathrm{H}(\mathrm{s})$ is:

$$
\mathcal{L}^{-1}[\mathrm{H}(s)]=\mathcal{L}^{-1}\left[\frac{s}{s+\alpha}\right]=\delta(t)-\alpha e^{-\alpha s}
$$

Where $\alpha$ is the inverse decay time: $\alpha \equiv 1 / \tau_{\text {Decay Time }}$. In the discrete-time domain, this result is:

$$
\mathrm{H}(t)=\delta[n]-\alpha e^{-\alpha n \Delta t}
$$

Alternatively, the entire result can be derived using the $Z$ transform. Starting with the (very) basic thermal equilibration, finite difference equation:

$$
T_{o}[n]-\frac{T_{o}[n-1]}{1+\Delta t / \tau_{\text {Decay }}}=\frac{T_{H B}[n]-T_{H B}[n-1]}{1+\Delta t / \tau_{\text {Decay }}}
$$

We Z-transform both sides of this equation and solve for the response temperature as a function of the heat bath temperature, $T_{o} / T_{H B}$ :

$$
H(z)=\frac{1-z^{-1}}{1-z^{-1}+\frac{\Delta t}{\tau_{\text {Decay }}}}
$$

Define the parameter $\alpha$ as:

$$
\alpha \equiv\left[1+\frac{\Delta t}{\tau_{\text {Decay }}}\right]^{-1}
$$

Then $H(z)$ can be written as:

$$
H(z)=\frac{\alpha\left(1-z^{-1}\right)}{1-\alpha z^{-1}}
$$


The inverse Z-transform of $\mathrm{H}(z)$, Eq. 10, is

$\mathrm{H}(n)=\delta(n)-(1-\alpha) \alpha^{n}$

which is functionally identical to Eq. 6 . Both are initial Kronecker delta functions followed by a decaying exponential. Equations 11 and 6 are plotted in Fig. 4 below.

Fig. 4. Time-domain system response function.

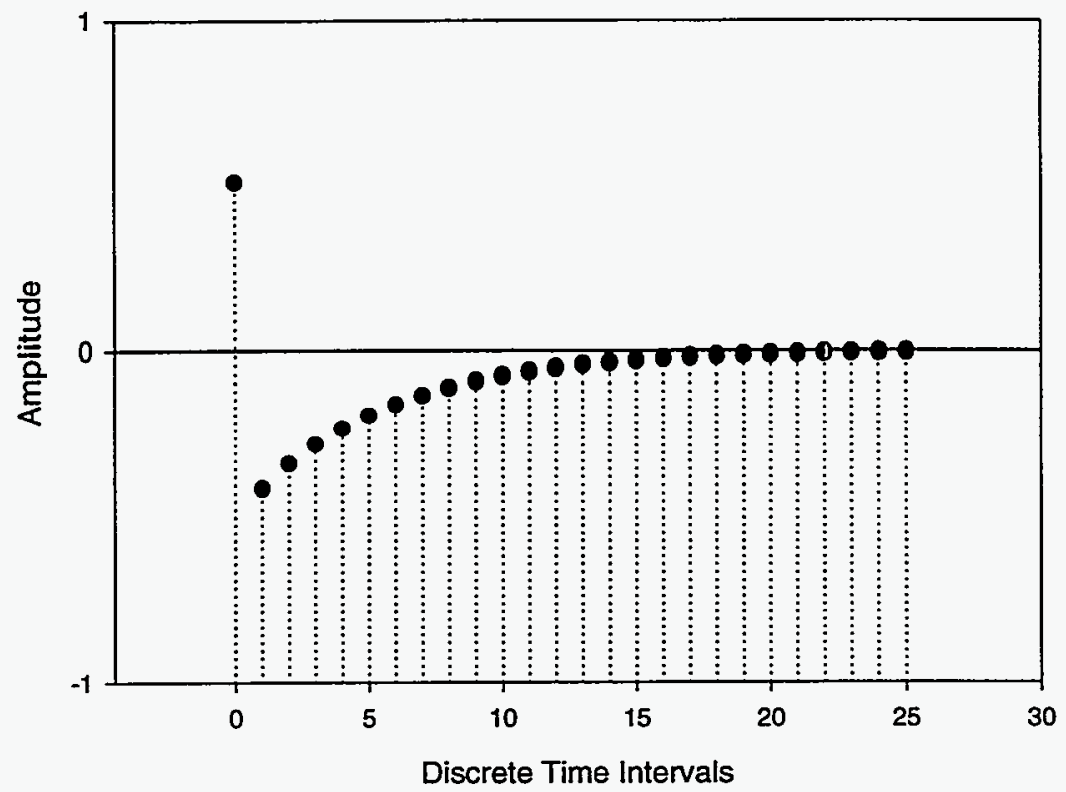

Equation 6 or 11 is then convolved with the compensation measurement following the standard formula:

$\mathrm{FP}_{\text {correct }}=\int_{\tau=-\infty}^{\tau=t} \mathrm{~T}_{\mathrm{FP}}(\tau) \mathrm{H}(t-\tau) d \tau$

In the discrete-time domain, the convolution integral becomes a sum:

$\mathrm{FP}_{\text {correct }}[n]=\sum_{i=0}^{i=n} \mathrm{~T}_{\mathrm{FP}}[i] \mathrm{H}[n-i]$

\section{PROCEDURE}

The procedure for correcting the temperature fluctuations of the heat bath is outlined in the flow chart below, Fig. 5. First, the decay time of the calorimeter is measured during the equilibration phase. From this information the system response is constructed according to Eq. 11 above, including the possibility of multiple exponentials. The result is subtracted from the differential temperature measurement to correct for the fluctuations in the heat-bath temperature.

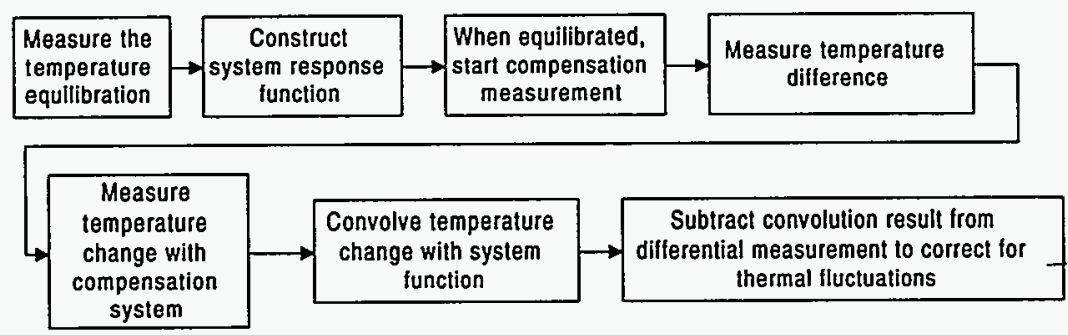


Fig. 5. Procedure for compensating the temperature fluctuations of the heat bath using the compensation measurement.

\section{THEORETICAL RESULTS}

We tested this procedure using the simulation described above. The test function consisted of a random signal superposed with a sinusoid - the same function that was illustrated in Fig. 3. The response of the calorimeter was modeled with the same (admittedly) simple finite difference equation discussed above. We assumed that the differential measurement accurately measures the thermal difference across the thermal resistance, and the compensation measurement accurately measures the heat-bath temperature (relative to an arbitrary standard). Therefore, the temperature plots for the difference and heat-bath temperatures in Fig. 3 also correspond to these measurements. We then convolved the simulated compensation signal from Fig. 3 with the system response function, Eq. 11, using the convolution sum, Eq. 13. This result was subtracted from the simulated differential measurement. The results are shown in Fig. 6.

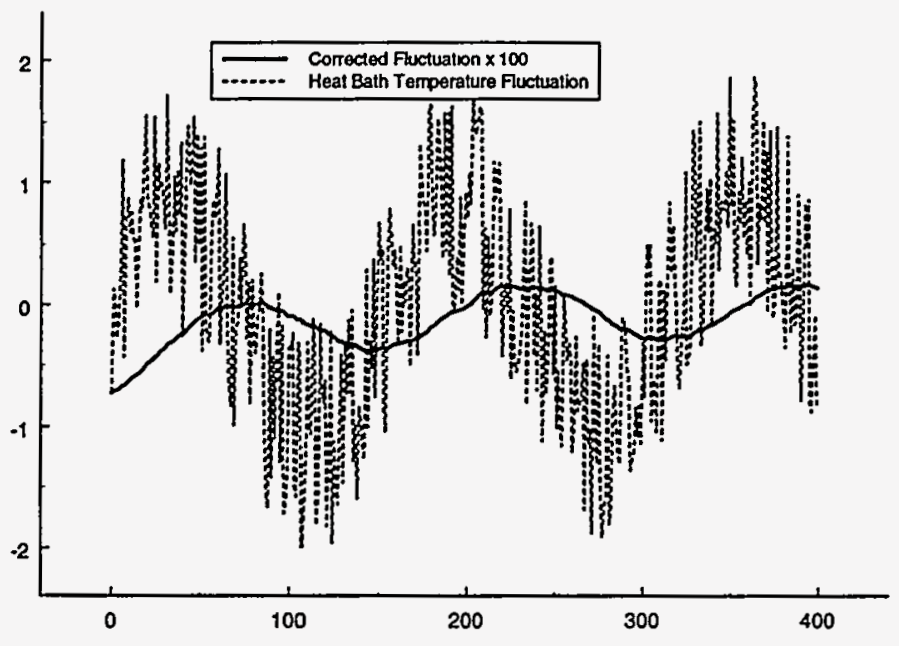

Fig. 6. Results of simulation of compensation method. Initial thermal fluctuations measured by the differential measurement are shown in first plot. Corrected temperature measurements are shown magnified by a factor of 100 in the second plot. The magnification was necessary because the compensation proved quite effective.

The results in Fig. 6 show that the compensation method is very effective, at least in the simulation. The effect of the temperature fluctuations are reduced approximately by a factor of 100 . This improvement will depend on several parameters, including the number of sample points in a nominal decay time.

\section{Experimental Results}

We tested this approach experimentally using a standard resistance-wire, bridge calorimeter that had extra temperature-sensing wires built in. The first step was to determine the calorimeter equilibration time for heat-bath temperature changes. When the calorimeter was in steady state, a step function in heat-bath temperature was introduced by pouring ice into the water bath. The calorimeter responded as expected, with a decaying exponential. Figure 7 plots these results. The heat-bath temperature changes as a definite step function and the calorimeter response is shown as a decaying exponential. We then fit a multi-pole model to the data using a Laplace-transform regression program. The model was of the form

$$
\frac{A s}{s+\alpha}+\frac{B s}{s+\beta},
$$


where $\alpha$ and $\beta$ are inverse time constants and $A$ and $B$ are amplitudes. From the fitted model we constructed the time-domain transfer function.

Calorimeter and Heat Bath Response

Fig.7. A plot of the heat-bath temperature and the calorimeter response as a function of time after ice is poured into the water bath. A step function is introduced in the heat-bath temperature.

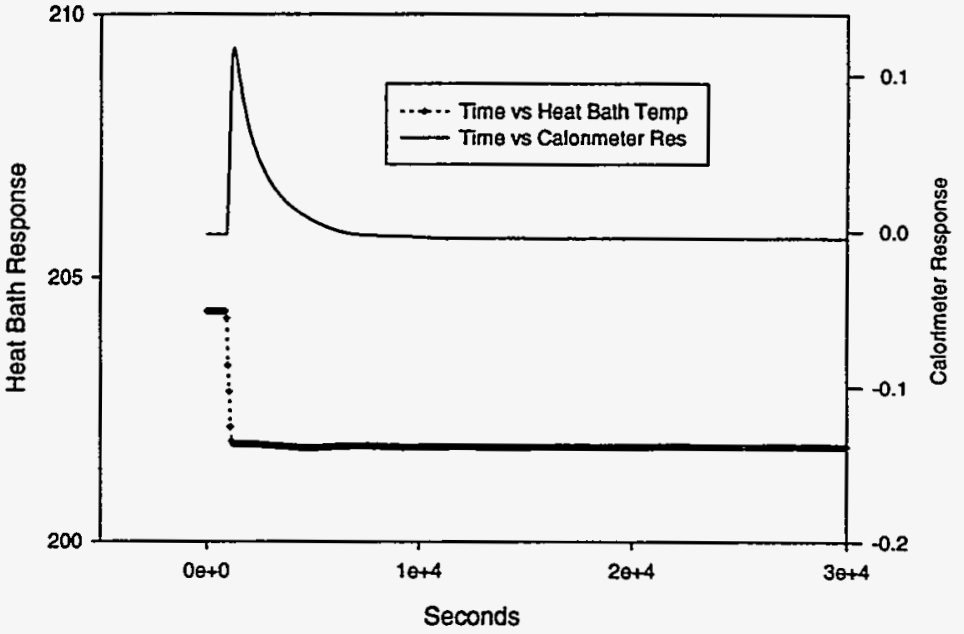

We then tested the compensation method using the time-domain transfer function. A $0.5-\mathrm{W}$ source was placed into the water bath calorimeter. The water-bath controller was turned off so that it would be more affected by ambient temperature fluctuations. The water bath should also change temperature from equilibration with the heat source. Fig. 8 plots the results. Both the raw calorimeter data (output from the thermal gradient) and the compensated assay signals are shown. Without the heat bath temperature control, the heat bath has temperature fluctuations from changes in the ambient temperature. Also in effect is the equilibration with the thermal source. In the compensated signal, however, the effect of the ambient changes is greatly attenuated (because the thermal design of the heat bath is not optimized for this, the compensation is not perfect).

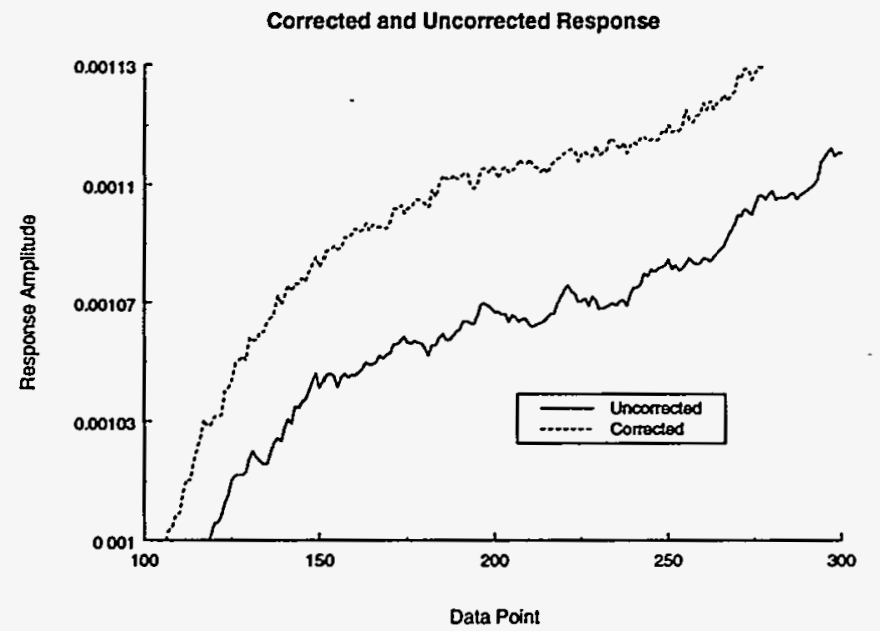

Fig. 8. A plot of the raw temperature measurement and the compensated value as a function of time. Note that compensation reduces temperature fluctuation. These data were taken prior to final equilibrium.

\section{OBSERVATIONS AND CONCLUSIONS}

1. This method does not have to be exceptionally precise. At present, there is essentially no compensation for the heat-bath temperature changes. If this method reduces-the effect of heatbath fluctuations by a factor of 10 (only $10 \%$ precision), it will be a significant improvement. Therefore, potential errors in fitting multiple exponentials are not very serious. 
2. The significance of this analysis is that the system response function is differential followed by a decaying exponential. Because the F-P measurement is differentiated and because it is a single fiber, this method essentially creates a "virtual" heat bath. When the F-P interferometer is started, the initial temperature is unknown. However, it has to have some temperature and whatever that temperature is, it is fixed at that instant in time. Because the reference temperature is at an instant in time, it cannot vary. This reference temperature is absolutely stable.

Subsequently, the F-P measures the temperature difference from this instant in time. Therefore, an absolute temperature reference is established.

3. This method can be used to compensate heat-bath fluctuations from both ambient changes and also equilibration to the sample.

4. Because there is no feedback system, there are no induced thermal fluctuations or instabilities.

\section{REFERENCES}

1. R. Rudy, S. Bayliss, D. S. Bracken, I. J. Bush, and P. Davis, "Fiber Optic Calorimetry," to be presented at the 39th Annual INMM Meeting, Naples, FL, July 26-30, 1998, Los Alamos National Laboratory document LA-UR-98-2840 (1998).

2. R. Rudy, S. C. Bayliss, D. S. Bracken, J. Bush, and P. Davis, "Fiber Optic Calorimetry," Los Alamos National Laboratory report LA-13392 (1998).

3. S. Krick, S. C. Bourret, P. R. Collinsworth, F. A. Duran, W. J. Hansen, W. C. Harker, K. E. Kroncke, and T. E. Sampson, "ARIES Calorimeter System Operators Manual," Los Alamos National Laboratory document LA-UR-98-1532 (1998).

4. R. Rudy and J. R. Wetzel, "Calorimeter Measurements of MOX," Los Alamos National Laboratory document LA-UR-98-1983 (1998).

5. Alan V. Oppenheim, Alan S. Willsky, and Ian T. Young, Signals and Systems, Prentice-Hall Signal Processing Series (Prentice-Hall, Englewood Cliffs, N.J. 1983) 